\title{
Tonal mapping of Xi'an Mandarin and Standard Chinese
}

\author{
Min Liu, ${ }^{1, a)}$ Yiya Chen, ${ }^{2, b)}$ and Niels O. Schiller ${ }^{2, b}$ \\ ${ }^{1}$ College of Chinese Language and Culture, Jinan University, 510610, Guangzhou, China \\ ${ }^{2}$ Leiden University Centre for Linguistics, Leiden University, Postbus 9515, 2300 RA, Leiden, The Netherlands
}

\begin{abstract}
:
One long-neglected fact in linguistic research on Standard Chinese (SC) is that most speakers of SC also speak a local dialect, which may share phonological features with SC. Tonal information can be a determinant of the phonological similarities or differences between some Chinese dialects and SC, yet relatively little empirical research has been conducted on the tonal system of other language varieties in Chinese aside from SC. Among these dialects, Xi'an Mandarin (XM) is particularly interesting for the seemingly simple, yet intricate mapping between its lexical tones with those in SC. In this study, the tonal systems of XM and SC were compared empirically. Tones with similar contours from XM and SC were paired, and both tone production and perception experiments were carried out on bidialectal speakers of XM and SC. The two experiments together showed that there is indeed systematic mapping of tones between XM and SC. The degree of similarity of the mapped tone pair in tone perception was largely dependent on the acoustic phonetic similarity between the tones in tone production, with a phonological rule playing a role in the tone pair of low contour. (C 2020 Acoustical Society of America. https://doi.org/10.1121/10.0000993
\end{abstract}

(Received 2 May 2019; revised 24 September 2019; accepted 16 October 2019; published online 30 April 2020)

[Editor: Richard A. Wright]

Pages: 2803-2816

\section{INTRODUCTION}

Chinese is a tonal language in which tones are used to distinguish lexical meanings. However, the term "Chinese" refers to a large number of Sinitic language varieties. While numerous studies have been conducted on Standard Chinese (SC; the official language of China), relatively little attention has been paid to other dialects or language varieties of Chinese. Some of the dialects differ from SC in both segmental and tonal information, whereas others, such as dialects within the Mandarin family, overlap largely in segmental information with SC. In these latter dialects, tonal information can be important as it determines the phonological similarities or differences between the dialect and SC.

In China, most speakers of SC speak a local dialect ( $\mathrm{Li}$ and Lee, 2004; Wiener and Ito, 2015). It is therefore of both practical and theoretical interest to systematically investigate the tonal similarities or differences between different dialects and SC. Such investigations can be the prerequisite to developing dialect-oriented speech synthesis and speech recognition technology (Czap and Zhao, 2017), guiding language pedagogy in teaching SC to dialectal speakers (Lam, 2005; Wong and Xiao, 2010), and addressing issues, such as whether the phonological information of one's two or more dialects are stored separately or integrally (Wu, 2015), or how cross-dialect phonological similarity/difference affects

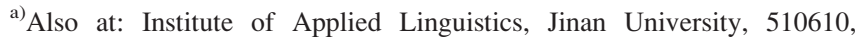
Guangzhou, China. Electronic mail: nwliumin@gmail.com

b) Also at: Leiden Institute for Brain and Cognition, Leiden University, Postbus 9515, 2300 RA, Leiden, The Netherlands.
}

lexical access in the minds of bidialectal tonal language speakers.

Currently, relatively little empirical research has been conducted on the tonal system of other language varieties except for SC; even less research has compared the tonal system of other language varieties with that of SC. As language varieties within the Mandarin family rely largely on tonal information to make distinctions from SC ( $\mathrm{Li}, 2017)$, the present study aimed to empirically compare the tonal systems of two closely related dialects in the Mandarin family, SC and Xi'an Mandarin (XM).

According to Chappell (2001), there are ten major dialect groups in Chinese (but see Yuan, 1989; Li and Thompson, 1981, which argue for seven major dialect groups). The Mandarin family is the largest Chinese dialect group. It contains a group of Chinese varieties, which are typically spoken in northern and southwestern China. The most influential language within the Mandarin family is SC. The other dialects within the Mandarin family share a common logographic writing system with SC and bear high resemblance with $\mathrm{SC}$ as to lexical items and syntactic forms (Cheng, 1991). Some dialects, such as XM, also exhibit a large overlap of segmental features with SC. More interestingly, the tones of XM seem to have a one-to-one correspondence with those of SC (Li, 2001; Zhang, 2009). This overall correspondence between the two tonal systems is quite unique and makes XM a very compelling case to study.

$\mathrm{XM}$ is a Mandarin dialect typically spoken in the urban areas of Xi'an, the capital of Shaanxi Province. It is the representative dialect of the Guanzhong dialect spoken in the Guanzhong area (Li and Stephen, 1987). XM directly 
originated from the official language in ancient China and has important historical value. As in SC, there are four tonal categories in XM, and they are referred to as T1, T2, T3, and $\mathrm{T} 4 .{ }^{1}$ Here, the terms T1-T4 are adopted to suggest that words which share the same tonal categories across the two dialects are etymologically related translation equivalents in most cases. Across XM and SC, different tones distinguish lexical meanings for syllables with the same segment. For example, the segment ma means mother, hemp, horse, and to scold, respectively, when it is combined with the four lexical tones (tonal category: T1, T2, T3, and T4). On the 5-point scale notation system (Chao, 1930, 1968), the pitch value of the SC tones has been established as 55 (T1), 35 (T2), 214 (T3), and 51 (T4). However, there have been discrepancies among researchers regarding the specific pitch value of each XM tone (see Table I for a summary of the representative transcriptions of XM tones).

The majority of the existing studies on XM tones, including the first six studies listed in Table I, have been based on impressionistic observation. Pitch values of XM tones in these studies could be susceptible to the subjective pitch sensitivity of the researchers. It is therefore not surprising that these studies vary in pitch value for each tonal category. The remaining studies, such as the last three in Table I, have attempted to study the pitch value of XM tones with more objective acoustic methods. However, these studies either sampled from a very limited number of speakers (e.g., two in Ma, 2005; one in Ren, 2012) or lacked control of lexical properties of the stimuli used (e.g., Zhang and Shi, 2009). It is not known to what degree these results can represent the typical tonal patterns of XM. The present study thus decided to empirically examine the acoustic properties of XM tones with a larger sample of speakers and stimuli and better control of lexical properties of the stimuli.

Although the specific pitch value of each tonal category in XM varies among previous studies, the basic tonal contour shape tends to be largely consistent across studies. Generally, the four tonal categories of XM possess the tonal contours of low-falling (T1), mid-rising (T2), high-falling (T3), and high-level (T4). Interestingly, XM tones display almost the same tonal contours as SC tones. In SC, tonal contours of the four tonal categories are described as high-level (T1, 55), mid-rising (T2, 35), low-falling-rising (T3, 214), and high-falling (T4, 51). As one can see, both

TABLE I. Representative transcription of XM tones in previous studies.

\begin{tabular}{lcccc}
\hline \hline Reference & T1 & T2 & T3 & T4 \\
\hline Bai (1954) & 21 & 24 & 453 & 45 \\
Luo and Wang (1981) & 31 & 24 & 42 & 55 \\
Yuan (1989) & 21 & 24 & 53 & 45 \\
Wang (1996) & 21 & 24 & 53 & 44 \\
Peking University (1989) & 21 & 24 & 53 & 55 \\
Sun (2007) & 31 & 24 & 53 & 55 \\
Ma (2005) & 21 & 24 & 53 & 44 \\
Zhang and Shi (2009) & 31 & 24 & 52 & 55 \\
Ren (2012) & 31 & 24 & 52 & 55 \\
\hline \hline
\end{tabular}

tonal systems of SC and XM contain tones of high-level, mid-rising, and high-falling tonal contours, and each of these tone pairs of similar contours share similar pitch values across the two tonal systems, although the similar contours do not necessarily represent the same tonal category in the two tonal systems (see Table II for details). Moreover, $\mathrm{SC}$ has a tone of low-falling-rising tonal contour, whereas $\mathrm{XM}$ has a tone of low-falling tonal contour without the rising tail. The former, however, would lose its rising tail when placed before other tones in SC (i.e., $\{\mathrm{T} 3[214]+\mathrm{T} 1 /$ $\mathrm{T} 2 / \mathrm{T} 4[55 / 35 / 51]\} \rightarrow\{\mathrm{T} 3[21]+\mathrm{T} 1 / \mathrm{T} 2 / \mathrm{T} 4[55 / 35 / 51]\}$; see Dow, 1972; Duanmu, 2007) and become similar to the latter. Overall, each XM tone seems to have a corresponding tone in SC with which it shares similar tonal contour and pitch value, resulting in a very interesting systematic mapping pattern between the tonal systems of XM and SC.

In fact, the mapping of tonal contours between the tonal systems of XM and SC has been proposed in previous studies. Li (2001) introduced the mapping pattern of XM tones and SC tones (similar as in Table II) and suggested that XM learners of SC utilize their knowledge of XM tones to produce SC tones. Zhang (2009) also claimed the presence of a comparable tonal contour for each XM tone in SC. She further statistically compared the F0 contour of each XM tone with its SC counterpart of a similar tonal contour. The results showed that although the paired tones were similar in tonal contour, there were detailed acoustic differences. Specifically, the XM low-falling tone was different from the citation form SC low-falling-rising tone in contour shape; the XM rising tone was significantly lower than the SC rising tone in F0 height except in the early-middle part; the XM falling tone had a similar initial F0 height with but higher final F0 height than the SC falling tone, and the XM level tone had an overall lower F0 height than the SC level tone. Zhang (2009) has made an attempt to reveal the acoustic similarities and differences between the two tonal systems empirically. However, it did not include details on the design of the production experiment; therefore it is not clear how the tonal patterns were obtained, and the comparison did not seem to be made on comparable datasets. The present study thus decided to compare the F0 acoustic properties of XM tones and SC tones in a pairwise fashion with a more balanced and comparable design. Since there have also been studies which declared that the duration of XM tones is overall shorter than that of SC tones (Guo et al., 2011), we compared both the F0 and duration for each pair of tones between SC and XM.

In addition to establishing the acoustic similarities or differences between the paired tones of XM and SC in production, we were also interested to know whether each tone pair of similar contours from XM and SC is perceived to be similar or different in tone perception by bidialectal speakers of XM and SC. The tone production and perception experiments together were expected to confirm the mapping pattern of the two tonal systems. So far, there have not been any perception studies comparing XM tones and SC tones. Conventionally, tone discrimination relies on several 
TABLE II. Paired tones with similar contours from SC and XM.

\begin{tabular}{|c|c|c|c|c|c|c|}
\hline \multirow[b]{2}{*}{ Tone pair } & \multicolumn{3}{|c|}{$\mathrm{SC}$} & \multicolumn{3}{|c|}{$\mathrm{XM}$} \\
\hline & Tonal category & Pitch value & Example & Tonal category & Pitch value & Example \\
\hline Level contour & SC_T1 & 55 & ma1/妈 & XM_T4 & $55 / 44 / 45$ & $\mathrm{ma} 4 /$ 骂 \\
\hline Rising contour & SC_T2 & 35 & ma2/麻 & XM_T2 & 24 & $\mathrm{ma} 2 /$ 麻 \\
\hline Low contour & SC_T3 & 214 & ma3/马 & XM_T1 & $21 / 31$ & $\mathrm{mal} /$ 妈 \\
\hline Falling contour & $\mathrm{SC} \_\mathrm{T} 4$ & 51 & ma4/骂 & XM T3 & $52 / 53 / 42$ & $\mathrm{ma} 3 /$ 马 \\
\hline
\end{tabular}

perceptual cues, among which the most widely adopted and important perceptual cues have proved to be F0 height and F0 contour, according to previous cross-language studies (Gandour, 1983, 1984; Gandour and Harshman, 1978; Francis et al., 2008). The relative importance of these two cues, however, varies among listeners of different language backgrounds. SC listeners tended to attach more importance to F0 contour than F0 height, whereas Cantonese and English listeners gave more weight to F0 height than F0 contour (Gandour, 1983, 1984). Apart from the F0-related features, other acoustic properties, such as duration, amplitude contour, and voice quality, have also been shown to serve as secondary cues for tone discrimination, especially when the primary F0 information was not available (Liu and Samuel, 2004; Whalen and Xu, 1992; Yang, 2015). Furthermore, phonological rules might play a role in tone discrimination. For example, SC native listeners found it more difficult to discriminate between the rising tone and the low-falling-rising tone than other tone pairs in SC (Huang, 2012), which was partly attributed to the tone sandhi rule that makes the two tones conditioned allophonic tonal variants. Specifically, the low-falling-rising tone would be realized as a rising tone when it precedes another low-falling-rising tone (Duanmu, 2007). The native phonological rule can sometimes even affect tone discrimination in a non-native language. For example, Cantonese listeners with or without SC experience had difficulty distinguishing between the SC high-level tone (55) and high-falling tone (51; Hao, 2012; So and Best, 2010). This is because in Cantonese the high-level tone (55) has a free allophonic tonal variant, high-falling tone (53; Bauer and Benedict, 1997; Hashimoto, 1972; Yip, 2002; Yu, 2007), which shows phonetic similarity to the SC high-falling tone. In this study, based on the acoustic results in the tone production experiment, we ran a tone perception experiment to see whether each tone pair would be perceived as similar or different by the bidialectal speakers and how the acoustic differences in each tone pair affect tone perception.

To sum up, in the present study, tonal categories with similar contours from XM and SC were paired as in Table II. Both tone production and perception experiments were carried out to test whether each pair of tones is acoustically and perceptually similar or different. In experiment 1 , we compared the acoustic properties of the paired SC and XM tones produced by a group of highly proficient bidialectal speakers of these two dialects and established the acoustic differences of each tone pair. In experiment 2, we further investigated whether each tone pair would be perceived as similar or different by the bidialectal speakers of SC and $\mathrm{XM}$ and how the acoustic differences in each tone pair affect tone perception with a five-scale tone judgment task. Results from both experiments were expected to reveal the tonal similarity and confirm the mapping pattern of the two tonal systems.

\section{EXPERIMENT 1}

\section{A. Method}

\section{Participants}

Thirty bidialectal speakers of SC and XM (16 males, 14 females) were selected and paid to participate in the experiment. All the selected participants achieved the 1B level in the Putonghua Shuiping Ceshi (National Standard Chinese Test), indicating that they have native proficiency in SC without regional accents. Their XM reached a comparable high proficiency level, judging from their performance on a story reading task and self-reported language proficiency through an adapted version of the LEAP-Q questionnaire (Marian et al., 2007). The participants acquired both dialects before the age of 6 years old and were early XM_SC bidialectal speakers with the first dialect (D1) being either XM or SC. All of them were born and raised in the urban areas of Xi' an and had no living experience outside of Xi'an. The participants were all undergraduate or graduate students at local universities. Their age ranged from 19 to 28 years old $(M \pm \mathrm{SD}: 22.5 \pm 3.2)$. None of them had reported any speech or hearing disorders.

\section{Stimuli}

Thirty monosyllabic minimal tone sets with full sets of all four tones were selected. The four monosyllables within one minimal tone set were distinguished merely by tone with the segments being identical. An exemplar of a full minimal tone set was mal, ma2, ma3, and ma4. The complete list can be found in Table III. The monosyllables were selected on the condition that no pronunciation difference exists for the segment of each syllable between SC and XM, to avoid any potential effect of segmental pronunciation difference on tones. The monosyllabic items are frequent monosyllabic words with more than 4500 occurrences in a corpus of $193 \times 10^{6}$ words ( $\left.\mathrm{Da}, 2004\right)$. Within each minimal tone set, the monosyllabic words have comparable word frequencies. In total, 120 monosyllabic words (30 syllables $\times 4$ 
TABLE III. The full monosyllabic words used in the two experiments.

\begin{tabular}{|c|c|c|c|c|}
\hline \multirow{2}{*}{$\begin{array}{l}\text { Syllable } \\
\text { number }\end{array}$} & \multicolumn{4}{|c|}{ Tone } \\
\hline & $\mathrm{T} 1$ & $\mathrm{~T} 2$ & $\mathrm{~T} 3$ & $\mathrm{~T} 4$ \\
\hline 1 & 逼 (bi1, to force) & 鼻 (bi2, nose) & 比 (bi3, to compare) & 闭 (bi4, to close) \\
\hline 2 & 搭 (da1, to travel by) & 达 (da2, to reach) & 打 (da3, to hit) & 大 (da4, big) \\
\hline 3 & 低 (di1, low) & 敌 (di2, enemy) & 底 (di3, bottom) & 弟 (di4, younger brother) \\
\hline 4 & 督 (du1, to supervise) & 毒 (du2, poison) & 奢 (du3, to gamble) & 度 (du4, degree) \\
\hline 5 & 涛 (tao1, great waves) & 桃 (tao2, peach) & 讨 (tao3, to ask for) & 套 (tao4, case) \\
\hline 6 & 梯 (ti1, ladder) & 题 (ti2, question) & 体 (ti3, body) & 替 (ti4, to substitute for) \\
\hline 7 & 通 (tong1, to go through) & 铜 (tong2, copper) & 桶 (tong3, bucket) & 痛 (tong4, pain) \\
\hline 8 & 方 (fang1, square) & 房 (fang2, house) & 访 (fang3, to visit) & 放 (fang4, to put) \\
\hline 9 & 风 (feng1, wind) & 冯 (feng2, a surname) & 讽 (feng3, irony) & 凤 (feng4, phoenix) \\
\hline 10 & 呼 (hu1, to call) & 湖 (hu2, lake) & 虎 (hu3, tiger) & 户 (hu4, household) \\
\hline 11 & 灰 (hui1, grey) & 回 (hui2, to go back) & 毁 (hui3, to destroy) & 会 (hui4, be able to) \\
\hline 12 & 吸 (xi1, to absorb) & 席 (xi2, mat) & 洗 (xi3, to wash) & 戏 (xi4, drama) \\
\hline 13 & 歇 (xie1, to rest) & 邪 (xie2, evil) & 写 (xie3, to write) & 谢 (xie4, to thank) \\
\hline 14 & 星 (xing1, star) & 形 (xing2, shape) & 醒 (xing3, to wake up) & 姓 (xing4, surname) \\
\hline 15 & 虚 (xu1, virtual) & 徐 (xu2, slowly) & 许 (xu3, a surname) & 续 (xu4, to continue) \\
\hline 16 & 抽 (chou1, to pump) & 仇 (chou2, hatred) & 丑 (chou3, ugly) & 臭 (chou4, smelly) \\
\hline 17 & 鸡 (ji1, chicken) & 急 (ji2, urgent) & 挤 (ji3, crowded) & 记 (ji4, to remember) \\
\hline 18 & 街 (jie1, street) & 杰 (jie2, hero) & 姐 (jie3, elder sister) & 戒 (jie4, to quit) \\
\hline 19 & 居 (ju1, to reside) & 局 (ju2, bureau) & 举 (ju3, to lift) & 句 (ju4, sentence) \\
\hline 20 & 期 (qi1, period) & 旗 (qi2, flag) & 起 (qi3, to rise) & 气 (qi4, gas) \\
\hline 21 & 轻 (qing1, light) & 晴 (qing2, sunny) & 请 (qing3, to invite) & 庆 (qing4, to celebrate) \\
\hline 22 & 妈 (ma1, mom) & 麻 (ma2, hemp) & 马 $(\mathrm{ma} 3$, horse $)$ & 骂 (ma4, to scold) \\
\hline 23 & 汪 (wang1, a surname) & 王 (wang2, king) & 网 (wang3, network) & 旺 (wang4, prosperous) \\
\hline 24 & 屋 (wu1, house) & 吴 (wu2, a surname) & 五 (wu3, five) & 误 (wu4, mistake) \\
\hline 25 & 优 (you1, excellent) & 油 (you2, oil) & 有 (you3, to have) & 右 (you4, right) \\
\hline 26 & 威 (wei1, prestige) & 围 (wei2, to surround) & 伟 (wei3, great) & 胃 (wei4, stomach) \\
\hline 27 & 敲 (qiao1, to knock) & 桥 (qiao2, bridge) & 巧 (qiao3, skillful) & 俏 (qiao4, pretty) \\
\hline 28 & 荒 (huang1, uncultivated) & 黄 (huang2, yellow) & 谎 (huang3, lies) & 晃 (huang4, to sway) \\
\hline 29 & 秃 (tu1, bald) & 图 (tu2, picture) & 土 (tu3, dust) & 兔 (tu4, rabbit) \\
\hline 30 & 汤 (tang1, soup) & 唐 (tang2, Tang dynasty) & 躺 (tang3, to recline) & 菏 (tang4, scalding) \\
\hline
\end{tabular}

tones) were selected. Some disyllabic words were added as fillers.

\section{Recording}

The recordings took place in a soundproof booth of the behavioral laboratory at Shaanxi Normal University in Xi'an. Stimuli were randomly presented to the speakers with Eprime 2.0 (Psychology Software Tools, Sharpsburg, PA). Each speaker produced all the items in both SC and XM with no repetition in two separate sessions. The order of the sessions was counterbalanced. Half of the speakers did the SC session first and then the XM session, and the other half started with the XM session. Each session included one practice block and three experimental blocks. Between each block, there was a 3-min break. The practice block contained eight trials, which were not used in the experimental blocks, to familiarize the participants with the specific language mode. An experimental trial started with a $300 \mathrm{~ms}$ fixation cross, followed by a $200 \mathrm{~ms}$ pause. After that, a stimulus in the form of simplified Chinese character was presented on the screen. Speakers were requested to produce the stimulus in that particular language of the session in a self-paced fashion. They pressed a button to proceed to the next stimulus when finished producing the current stimulus. The interstimulus interval was $500 \mathrm{~ms}$. Instructions were given to the speakers visually on the screen in simplified Chinese characters and orally by the experimenter in that particular language before each session. All the stimuli were recorded at 16-bit resolution with a sampling rate of $44.1 \mathrm{kHz}$ on a laptop via an external digitizer (UA-1G, Cakewalk, Boston, MA). Altogether, 240 monosyllabic items (30 syllables $\times 4$ tones $\times 2$ languages) were elicited from each of the 30 speakers.

\section{Data analysis}

The F0 and duration of the speech items were analyzed. All the stimuli were manually annotated in Praat (Boersma and Weenink, 2015). A custom-made script was then used to extract ten equally distanced F0 values from the rhyme part of each time-normalized syllable. Gross errors in F0 extractions were manually corrected afterward. To eliminate between-speaker acoustic differences, the raw F0 values were transformed to $Z$-score ${ }^{2}$ for each speaker (Rose, 1987), pooling the SC and XM productions.

Statistical analyses of F0 were carried out using the growth curve analysis (Mirman, 2014) with the package lmerTest (Kuznetsova et al., 2017) in $R$ version 3.1.2 
(R Core Team, 2015). The overall F0 curves were modeled with up to second-order orthogonal polynomials, given that the most complex F0 contour in this study has a U-shaped curve. Three time terms of the models would be of interest: the intercept, the linear slope, and the steepness of the quadratic curvature, which indicate the overall F0 mean, the direction of F0 change such as rising or falling, and the steepness of F0 rising or falling, respectively. If tonal contours under investigation are different, we expect statistical difference in at least one of the three time terms. As we are interested in whether each pair of tones listed in Table II is acoustically similar or different, we built separate models for the F0 of each pair of tones. All the models included the random intercept of subjects on all time terms. The fixed effects of language (XM, SC) on all time terms, as well as the random intercept of items, and the random slope of subjects-by-language and items-by-language on all time terms were added in a stepwise fashion, and their effects on model fits were evaluated via model comparisons based on log-likelihood ratios.

Statistical analyses of duration were performed using linear mixed-effects regression models with the package lmerTest (Kuznetsova et al., 2017) in $R$ version 3.1.2 (R Core Team, 2015). As for F0, we built separate models for the duration of each pair of tones. All the models first included random intercepts of subjects and items. The fixed effect of language and random slopes of subjects-bylanguage and items-by-language were added in a stepwise fashion, and their effects on model fits were evaluated via model comparisons based on log-likelihood ratios.

\section{B. Results}

\section{1. $F O$}

The statistical results for the models of F0 of each tone pair are listed in Table IV. Figure 1 presents the mean F0 (Z-score) contours of the four tones in SC and XM. We report the F0 for each pair of tones of similar tonal contours in what follows.

a. Level contour: SC_T1 vs XM_T4. Results showed that the effect of language on the intercept did not improve model fit $\left[\chi^{2}(1)=0.03, p=0.87\right]$, nor did the effect of language on the linear term $\left[\chi^{2}(1)=1.54, p=0.21\right]$ and the effect of language on the quadratic term $\left[\chi^{2}(1)=0.23\right.$, $p=0.63]$. Overall, it seems that the F0 contours of SC_T1 and XM_T4 did not differ from each other.

b. Rising contour: SC_T2 vs XM_T2. Results showed that the effect of language on the intercept did not improve model fit $\left[\chi^{2}(1)=3.17, p=0.07\right]$, nor did the effect of language on the linear term $\left[\chi^{2}(1)=2.73, p=0.10\right]$. The effect of language on the quadratic term, however, did improve model fit $\left[\chi^{2}(1)=14.05, p<0.001\right]$. Therefore, the overall $\mathrm{F} 0$ mean and the direction of $\mathrm{F} 0$ rising did not differ between the two rising tones from SC and XM, but their steepness of rising differed with XM_T2 having a shallower curvature than SC_T2 $(\beta=-0.08, t=-4.01, p<0.001)$.

c. Low contour: SC_T3 vs XM_T1. The analyses of SC_T3 and XM_T1 data showed that the effect of language

TABLE IV. Summary of mixed-effects models for F0 of each tone par in experiment 1 .

\begin{tabular}{|c|c|c|c|c|c|}
\hline Tone pair & Effect type & Predictors & Degrees of freedom & $\chi^{2}$ & $p$-value \\
\hline \multirow[t]{6}{*}{ Level contour (SC_T1 vs XM_T4) } & \multirow[t]{3}{*}{ Fixed effects } & Language & 1 & 0.03 & 0.87 \\
\hline & & ot1: Language & 1 & 1.54 & 0.21 \\
\hline & & ot2: Language & 1 & 0.23 & 0.63 \\
\hline & \multirow[t]{3}{*}{ Random effects } & ot $1+$ ot $2 \mid$ Subjects & 6 & 1225.40 & $<0.001$ \\
\hline & & ot $1+$ ot $2 \mid$ Items & 6 & 2115.50 & $<0.001$ \\
\hline & & ot $1+$ ot $2 \mid$ Subjects: Language & 6 & 5834.30 & $<0.001$ \\
\hline \multirow[t]{7}{*}{ Rising contour (SC_T2 vs XM_T2) } & \multirow[t]{3}{*}{ Fixed effects } & Language & 1 & 3.17 & 0.07 \\
\hline & & ot1: Language & 1 & 2.73 & 0.10 \\
\hline & & ot2: Language & 1 & 14.05 & $<0.001$ \\
\hline & \multirow[t]{4}{*}{ Random effects } & ot $1+$ ot $2 \mid$ Subjects & 6 & 2843.70 & $<0.001$ \\
\hline & & ot $1+$ ot $2 \mid$ Items & 6 & 3047.00 & $<0.001$ \\
\hline & & ot $1+$ ot $2 \mid$ Subjects: Language & 6 & 3113.60 & $<0.001$ \\
\hline & & ot $1+$ ot 2 |Items: Language & 6 & 121.19 & $<0.001$ \\
\hline \multirow[t]{6}{*}{ Low contour (SC_T3 vs XM_T1) } & \multirow[t]{3}{*}{ Fixed effects } & Language & 1 & 57.85 & $<0.001$ \\
\hline & & ot1: Language & 1 & 26.79 & $<0.001$ \\
\hline & & ot2: Language & 1 & 37.58 & $<0.001$ \\
\hline & \multirow[t]{3}{*}{ Random effects } & ot $1+$ ot $2 \mid$ Subjects & 6 & 1021.00 & $<0.001$ \\
\hline & & ot $1+$ ot $2 \mid$ Items & 6 & 6993.40 & $<0.001$ \\
\hline & & ot $1+$ ot $2 \mid$ Subjects: Language & 6 & 2046.10 & $<0.001$ \\
\hline \multirow[t]{6}{*}{ Falling contour (SC_T4 vs XM_T3) } & \multirow[t]{3}{*}{ Fixed effects } & Language & 1 & 9.06 & 0.003 \\
\hline & & ot1: Language & 1 & 0.50 & 0.48 \\
\hline & & ot2: Language & 1 & 0.83 & 0.36 \\
\hline & \multirow[t]{3}{*}{ Random effects } & ot $1+$ ot $2 \mid$ Subjects & 6 & 2507.30 & $<0.001$ \\
\hline & & ot $1+$ ot $2 \mid$ Items & 6 & 2211.00 & $<0.001$ \\
\hline & & ot1 + ot2|Subjects: Language & 6 & 2923.10 & $<0.001$ \\
\hline
\end{tabular}




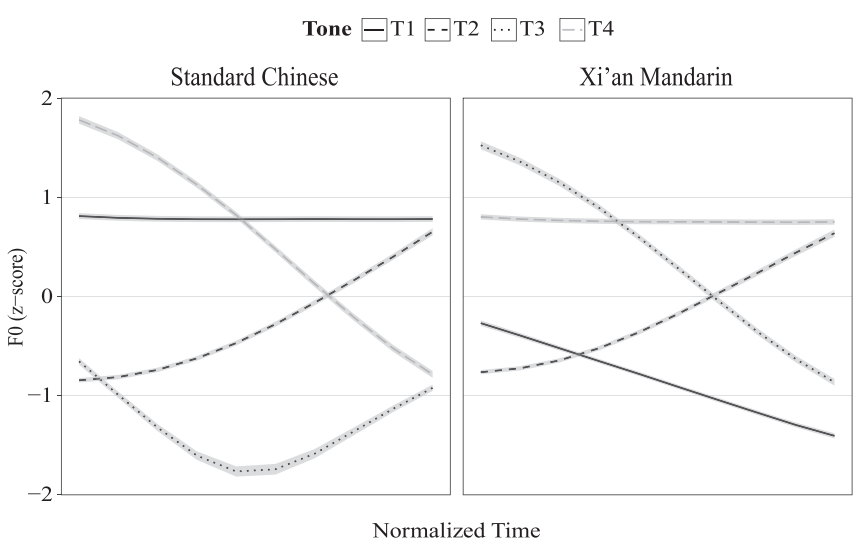

FIG. 1. Mean F0 (Z-score) contours of the four tones in SC and XM. The F0 values of each tone were averaged over 30 speakers and 30 monosyllabic items with the tone of each item represented by 10 equally distanced F0 values taken from the rhyme part of the time-normalized item. The grey areas indicate the $95 \%$ confidence interval of the corresponding mean.

on the intercept significantly improved model fit $\left[\chi^{2}(1)\right.$ $=57.85, p<0.001]$, as well as the effect of language on the linear term $\left[\chi^{2}(1)=26.79, p<0.001\right]$ and the effect of language on the quadratic term $\left[\chi^{2}(1)=37.58, p<0.001\right]$. Apparently, SC_T3 was different from XM_T1 in all three time terms. The overall F0 mean of XM_T1 was significantly higher than SC_T3 $(\beta=0.46, t=9.13, p<0.001)$. The direction of the F0 change was also different between the two tones $(\beta=-0.96, t=-14.06, p<0.001)$, with SC_T3 having a falling-rising contour and XA_T1 having a low-falling contour without the rising tail. Moreover, the F0 curvature of XA_T1 was shallower than that of SC_T3 $(\beta=-1.08, t=-14.23, p<0.001)$.

d. Falling contour: SC_T4 vs XM_T3. The analyses of SC_T4 and XM_T3 showed that there was a significant effect of language on the intercept $\left[\chi^{2}(1)=9.06, p=0.003\right]$. However, no language effect on the linear term $\left[\chi^{2}(1)=0.50\right.$, $p=0.48]$ or the quadratic term $\left[\chi^{2}(1)=0.83, p=0.36\right]$ was found. Evidently, the overall F0 mean of XM_T3 was lower than that of SC_T4 $(\beta=-0.19, t=-3.08, p=0.002)$. The direction of F0 falling and the steepness of F0 falling were not significantly different between the two falling tones.

\section{Duration}

The statistical results for the models of duration of each tone pair are listed in Table V. Figure 2 presents the mean durations of the four tones in SC and XM. The following reports the duration results for each pair of tones of similar tonal contours.

a. Level contour: SC_T1 vs XM_T4. There was a significant main effect of language $\left[\chi^{2}(1)=10.91, p<0.001\right]$. SC_T1 was significantly longer $(45.09 \mathrm{~ms})$ than XM_T4.

b. Rising contour: SC_T2 vs XM_T2. The effect of language significantly improved model fit $\left[\chi^{2}(1)=425.36\right.$, $p<0.001]$. SC_T2 was $47.60 \mathrm{~ms}$ longer than XM_T2.

c. Low contour: SC_T3 vs XM_T1. Not surprisingly, a significant main effect of language was also found for the durations of this tone pair $\left[\chi^{2}(1)=71.33, p<0.001\right]$. SC_T3 was considerably longer than XM_T1 with the duration difference reaching up to $166.29 \mathrm{~ms}$.

d. Falling contour: SC_T4 vs XM_T3. An investigation of the durations of the tone pair of the falling contour revealed no effect of language $\left[\chi^{2}(1)=0.64, p=0.42\right]$, indicating that there was no duration difference between SC_T4 and XM_T3.

From the above comparisons of F0 and duration for each pair of tones of similar tonal contours, the acoustic patterns of each tone pair can be summarized as follows (see also Table VI). First, the tone pair of level contour did not show any difference in F0. However, the duration of the tone of level contour in $\mathrm{SC}$ was significantly longer than that of its counterpart in XM. Second, the overall F0 mean and the direction of F0 change did not differ between the two tones of rising contour in SC and XM, despite a shallow curvature of the rising F0 contour in XM_T2 relative to SC_T2. In addition, the duration of XM_T2 was considerably shorter than that of SC_T2. Third, the two tones of low contour in $\mathrm{SC}$ and XM were significantly different from each other regarding the overall F0 mean, the direction of F0 change, and the steepness of F0 change. In fact, their contour

TABLE V. Summary of mixed-effects models for the duration of each tone pair in experiment 1.

\begin{tabular}{|c|c|c|c|c|c|}
\hline Tone pair & Effect type & Predictors & Degrees of freedom & $\chi^{2}$ & $p$-value \\
\hline \multirow[t]{3}{*}{ Level contour (SC_T1 vs XM_T4) } & Fixed effects & Language & 1 & 10.91 & $<0.001$ \\
\hline & Random effects & $1 \mid$ Items & 1 & 1332.20 & $<0.001$ \\
\hline & & $1+$ Language $\mid$ Subjects & 2 & 155.75 & $<0.001$ \\
\hline \multirow[t]{3}{*}{ Rising contour (SC_T2 vs XM_T2) } & Fixed effects & Language & 1 & 425.36 & $<0.001$ \\
\hline & Random effects & $1+$ Language $\mid$ Subjects & 2 & 182.79 & $<0.001$ \\
\hline & & $1+$ Language $\mid$ Items & 2 & 14.69 & $<0.001$ \\
\hline \multirow[t]{3}{*}{ Low contour (SC_T3 vs XM_T1) } & Fixed effects & Language & 1 & 71.33 & $<0.001$ \\
\hline & Random effects & $1 \mid$ Items & 1 & 2173.60 & $<0.001$ \\
\hline & & $1+$ Language $\mid$ Subjects & 2 & 308.28 & $<0.001$ \\
\hline \multirow[t]{3}{*}{ Falling contour (SC_T4 vs XM_T3) } & Fixed effects & Language & 1 & 0.64 & 0.42 \\
\hline & Random effects & $1 \mid$ Items & 1 & 1318.50 & $<0.001$ \\
\hline & & $1+$ Language Subjects & 2 & 165.27 & $<0.001$ \\
\hline
\end{tabular}




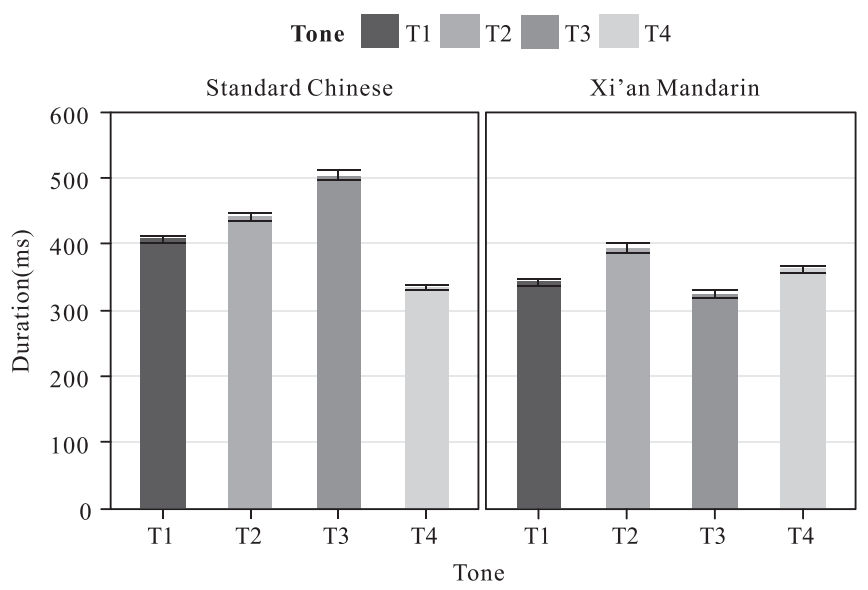

FIG. 2. Mean durations with the $95 \%$ confidence interval of the four tones in $\mathrm{SC}$ and $\mathrm{XM}$.

shape differed with the SC tone having a low-falling-rising contour and the XM tone having a low-falling contour without the rising tail. The former also tended to be remarkably longer than the latter. Fourth, having almost parallel F0 contours, the two tones of high-falling contour in SC and $\mathrm{XM}$ revealed differences in the overall $\mathrm{F} 0$ mean with an overall higher F0 contour of SC_T4 compared to XM_T3. Nevertheless, their durations did not differ.

\section{EXPERIMENT 2}

Having established the acoustic differences of each tone pair, the question arises as to whether the acoustic differences in each tone pair can be perceived. In fact, the two tonal systems provide an interesting test case for us to look into the relationship of the production and perception of tones by the bidialectal tonal language speakers. As shown in experiment 1, the F0 difference of each tone pair ranged from no F0 difference (level tone pair) through F0 curvature difference (rising tone pair) to F0 height difference (falling tone pair) and F0 contour difference (low tone pair). With this setup of the two tonal systems, we could investigate how different F0 dimensions affect tone perception of the bidialectal tone language speakers. In this session, a five-scale tone judgment task was adopted to examine whether the acoustic differences in each tone pair can be perceived. We then compared the tone perception results of different tone pairs to reveal how different F0 dimensions affect tone perception.

TABLE VI. Summary of the acoustic differences for each tone pair of SC and XM. ${ }^{\mathrm{a}}$

\begin{tabular}{lllll}
\hline \hline & \multicolumn{3}{c}{$\mathrm{F} 0$} \\
\cline { 2 - 3 } Tone pair & Intercept & Slope & Quadratic & $\begin{array}{c}\text { Duration } \\
\text { estimate }\end{array}$ \\
\hline Level (SC_T1 vs XM_T4) & n.s. & n.s. & n.s. & $* * *$ \\
Rising (SC_T2 vs XM_T2) & n.s. & n.s. & $* * *$ & $* * *$ \\
Low (SC_T3 vs XM_T1) & $* * *$ & $* * *$ & $* * *$ & $* * *$ \\
Falling (SC_T4 vs XM_T3) & $* *$ & n.s. & n.s. & n.s. \\
\hline \hline
\end{tabular}

${ }^{\text {a Note: }}{ }^{* *} p<0.01 ; * * * p<0.001$; n.s.: not significant.

\section{A. Method}

\section{Participants}

Another set of 30 XM_SC bidialectal speakers (11 males, 19 females) with high proficiency in both dialects were selected and paid to participate in the experiment. They all achieved the 1B level in the Putonghua Shuiping Ceshi (National Standard Chinese Test). All the selected participants acquired both dialects before the age of 6 years old and were early XM_SC bidialectal speakers with a D1 of either XM or SC. They were born and raised in the urban areas of Xi'an and had no living experience outside of Xi'an. All were undergraduate or graduate students at local universities between 18 and 29 years old $(M \pm$ SD: 21.2 \pm 2.6 ). None of them had reported any speech or hearing disorders. Informed consent was obtained from all the participants before the experiment.

\section{Stimuli}

The four pairs of tones in experiment 1 were used for tone judgment. Since the two tones in each tone pair were similar in tonal contour overall, two pairs of tones of distinct tonal contours were added as fillers to avoid potential response strategies. Each tone pair was tested with all 30 root monosyllables in experiment 1, resulting in 30 trials. The two speech items in a trial always share the same segment so that participants could focus on the tone judgment. For example, a SC_T1 monosyllable (“妈”, mal, mother) was paired with its corresponding XM_T4 monosyllable (“骂”, ma4, to scold).

Four speakers were recruited to record the stimuli for the perception experiment. They were all university students aged 20-28. Two native speakers (one male, one female) of $\mathrm{SC}$, who were born and raised in Beijing and had no knowledge of any other dialects, recorded the SC monosyllabic sounds. Likewise, two native speakers (one male, one female) of XM, who were born and raised in the urban area of Xi'an and had no living experience outside of Xi'an, recorded the XM monosyllabic sounds. Note that as it is impossible to find monolingual XM speakers nowadays, the two native speakers of XM also speak SC fluently. The recordings took place in Beijing for the Beijing speakers and in Xi'an for the Xi'an speakers. All of the speech items were recorded at 16-bit resolution with a sampling rate of $44.1 \mathrm{KHz}$.

To ensure that the two XM speakers' tone patterns were representative enough of $\mathrm{XM}$, we compared the acoustic properties of their tone patterns with those of the XM tone patterns in experiment 1 (see Figs. 3 and 4). Statistical analyses of F0 were carried out using the growth curve analysis. And statistical analyses of duration were performed using linear mixed-effects regression models. We found no statistical differences in F0 and duration. It was therefore confirmed that the two XM speakers' production of XM tone patterns were representative patterns of XM and suitable for the perception study. We also compared the acoustic 
Overall F0

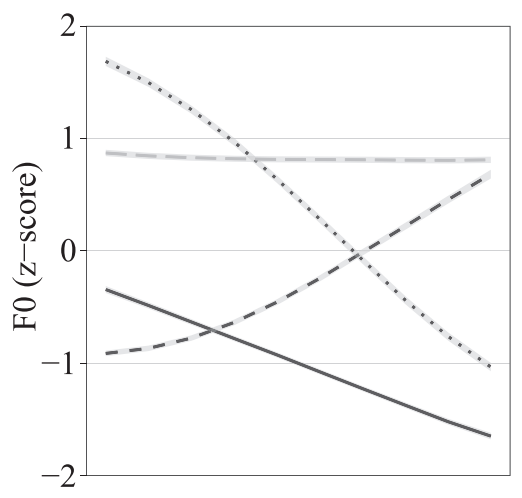

Male speaker's F0

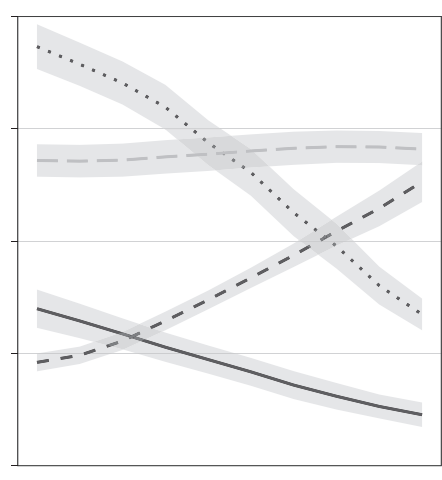

Normalized Time
Female speaker's F0

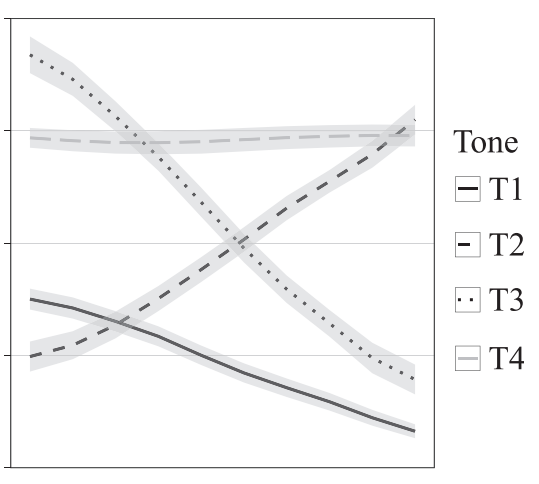

FIG. 3. Mean F0 (Z-score) contours of the four tones in XM by the $30 \mathrm{SC}$ and XM bidialectal speakers in experiment 1 (left), the male Xi'an speaker (middle), and the female Xi' an speaker (right) in experiment 2. The grey areas indicate the $95 \%$ confidence interval of the corresponding mean.

properties of the two SC speakers' tone patterns with those of the SC tone patterns in experiment 1 (see Figs. 5 and 6) and did not find statistical differences in F0 and duration either.

After normalizing the amplitude of all the speech items in Praat (Boersma and Weenink, 2015), we paired the Beijing female speaker's speech items with the Xi' an female speaker's corresponding speech items according to tone pairs. The same was done for the two male speakers' speech items. Instead of recording all the speech items by a XM_SC bidialectal speaker, we recorded the SC speech items by native speakers of SC and the XM speech items by native speakers of XM. This ensured more typical realizations of SC and XM tones. The inclusion of two groups of speakers could avoid potential speaker bias.

\section{Procedure}

Participants were tested individually in a soundproof booth of the behavioral laboratory at Shaanxi Normal University in Xi'an. All the trials (30 syllables $\times 6$ tone pairs $\times 2$ speaker groups) were randomly presented to the participants using the E-Prime 2.0 software (Psychology Software Tools, Sharpsburg, PA) through headphones at a comfortable listening level.

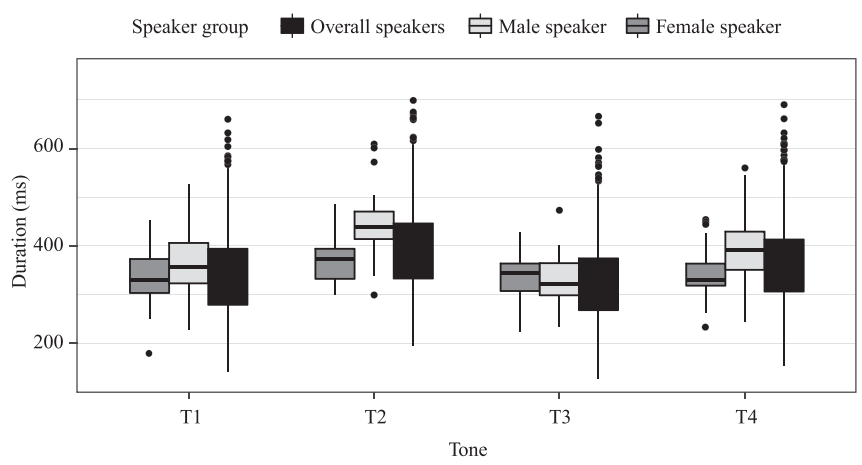

FIG. 4. Mean durations with the $95 \%$ confidence interval of the four tones in XM by the $30 \mathrm{SC}$ and XM bidialectal speakers in experiment 1 (black boxes), the male Xi'an speaker (light grey boxes), and the female Xi'an speaker (dark grey boxes) in experiment 2 .
The experiment included a practice block and four experimental blocks. The practice block contained six trials, which were not used in the experimental blocks. Each experimental block contained 90 trials. Between every second block, there was a 3-min break. An experimental trial started with a $100 \mathrm{~ms}$ warning beep, followed by a $300 \mathrm{~ms}$ pause. The first speech item was then presented. After a $600 \mathrm{~ms}$ pause, the second speech item was presented. The language order of the two speech items in a trial was counterbalanced for each speaker group of the trials. Half of the trials presented the SC item before its corresponding XM item, while the other half presented the SC item after its corresponding $\mathrm{XM}$ item. Participants were requested to judge the similarity of the two tones of the two speech items in a trial on a fivepoint scale, with " 1 " indicating "completely different" and "5" indicating "completely the same." Response accuracy rather than speed was stressed. However, if participants did not make any response from the onset of the second stimulus to $2.5 \mathrm{~s}$ after the offset of the second stimulus, the program moved on to the next trial automatically with an intertrial interval of $500 \mathrm{~ms}$. Instructions were given both visually on the screen and orally by the experimenter in SC before the experiment. To eliminate any influence of top-down knowledge on tone judgment, we did not mention the source languages of the auditory stimuli to the participants in the instructions.

\section{Data analysis}

To decide whether each pair of tones was perceived as similar or different, we analyzed the frequency distribution of the responses with the chi-square goodness-of-fit test. The observed frequency distribution of the responses was first compared with the expected frequency distribution (null hypothesis: equal proportions) for each tone pair. If the null hypothesis of equal proportions was rejected, the individual response category's contribution to the overall chi-square statistic was determined by calculating the square of the difference between the observed and expected frequencies for a category, divided by the expected frequency for that category. Generally speaking, categories with a larger difference between the observed and expected frequencies make a 
Overall SC F0

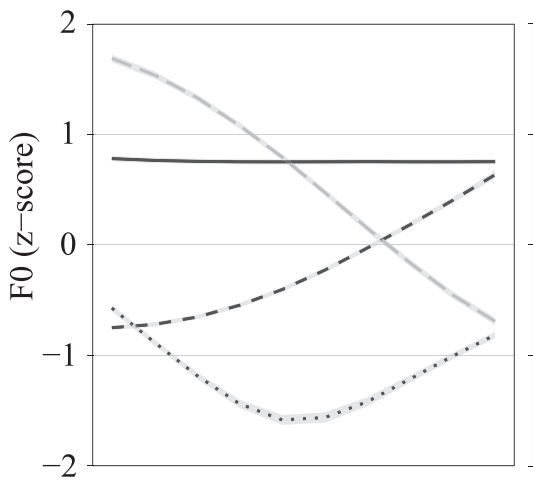

Beijing male speaker's F0

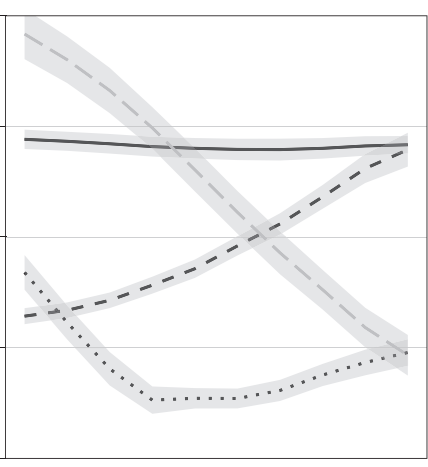

Beijing female speaker's F0

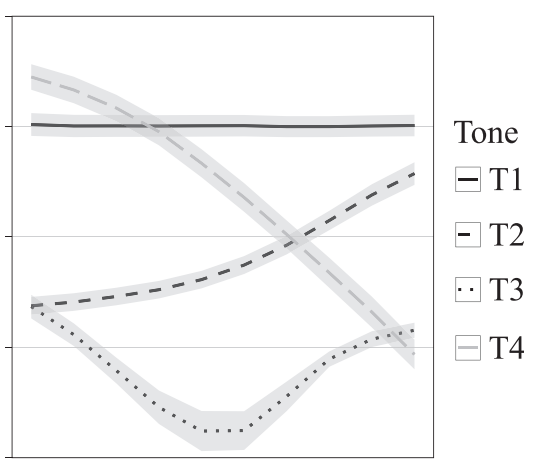

Normalized Time

FIG. 5. Mean F0 (Z-score) contours of the four tones in SC by the $30 \mathrm{SC}$ and XM bidialectal speakers in experiment 1 (left), the male Beijing speaker (middle), and the female Beijing speaker (right) in experiment 2 . The grey areas indicate the $95 \%$ confidence interval of the corresponding mean.

larger contribution to the overall chi-square statistic. After recognizing the response category that contributed the most to the overall chi-square statistic, we further conducted several pair-wise goodness-of-fit tests to compare this category's frequency with that of the other categories. If all the comparisons are statistically significant ( $p$-value adjusted), the category would be considered as the best indicator of the similarity/difference between the two tones under investigation.

The second analysis concerned how the varying acoustic differences of different tone pairs affect tone perception of XM_SC bidialectal speakers. All of the four pairs of tones were merged into one dataset, and the tone perception results of different tone pairs were compared. Statistical analyses were carried out with the package ordinal (Christensen, 2015) in $R$ version 3.1.2 (R Core Team, 2015). Cumulative link mixed models (CLMMs) were constructed for the dependent variable response $(1,2,3,4,5)$ with tone pair (level, rising, low, falling), language order (XM before SC; SC before XM), speaker group (female, male), listener gender (female, male) and their interactions as fixed factors, and subjects and items as random factors. The fixed factors were added in a stepwise fashion, and their effects on model fits were evaluated via model comparisons based on loglikelihood ratios. Post hoc pairwise comparisons between

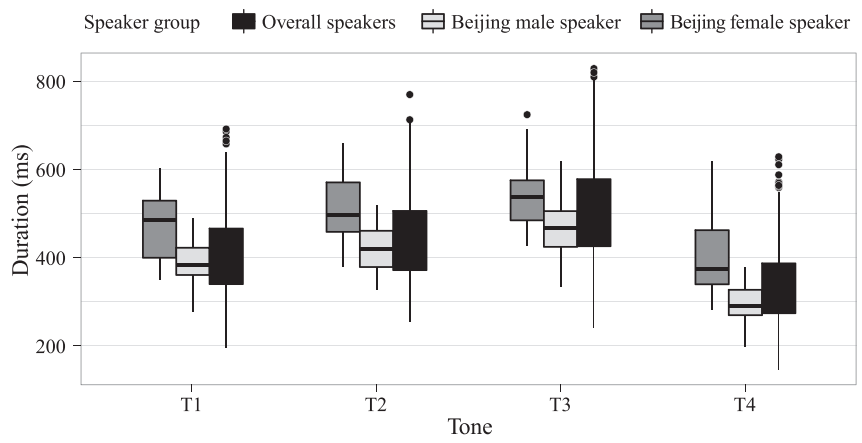

FIG. 6. Mean durations with the $95 \%$ confidence interval of the four tones in SC by the $30 \mathrm{SC}$ and XM bidialectal speakers in experiment 1 (black boxes), the male Beijing speaker (light grey boxes), and the female Beijing speaker (dark grey boxes) in experiment 2 . different tone pairs were conducted using the lsmeans package (Lenth, 2016) with single-step $p$-value adjustment.

\section{B. Results}

\section{Level contour: SC_T1 vs XM_T4}

The chi-square goodness-of-fit test showed that the responses were clearly not equally distributed $\left[\chi^{2}(4)\right.$ $=5634.42, p<0.001]$. As can be seen from Table VII, the response category 5 contributed the most to the overall chisquare statistic. Pairwise comparisons showed that the frequency of the response category 5 was significantly higher than that of the other categories (all $p$ 's $<0.001$ ), indicating that SC_T1 and XM_T4 were mostly judged as 5, i.e., completely the same.

\section{Rising contour: SC_T2 vs XM_T2}

The chi-square goodness-of-fit test showed that the responses were clearly not equally distributed $\left[\chi^{2}(4)\right.$ $=5677.38, p<0.001]$. Again, the response category 5 contributed the most to the overall chi-square statistic (see Table VII). Further pairwise comparisons showed that the frequency of the response category 5 was significantly higher than that of the other categories (all $p$ 's $<0.001$ ), indicating that SC_T2 and XM_T2 were mostly judged as 5, i. e., completely the same.

\section{Low contour: SC_T3 vs XM_T1}

The chi-square goodness-of-fit test showed that the responses were not equally distributed $\left[\chi^{2}(4)=1043.36\right.$, $p<0.001]$. As shown in Table VII, the response category 5 contributed the most to the overall chi-square statistic. However, the response category 1 also made a relatively large contribution to the overall chi-square statistic. Pairwise comparisons showed that the frequencies of the response categories 1 and 5 were significantly higher than those of the rest categories (all p's $<0.001$ ). Moreover, the frequency of the response category 5 was higher than that of the response category $1\left[\chi^{2}(1)=12.07, \quad p=0.0005\right]$. 
TABLE VII. Response counts for each tone pair. ${ }^{\text {a }}$

\begin{tabular}{|c|c|c|c|c|c|c|}
\hline \multirow[b]{2}{*}{ Tone pair } & \multirow[b]{2}{*}{ Measure } & \multicolumn{5}{|c|}{ Response category } \\
\hline & & 1 & 2 & 3 & 4 & 5 \\
\hline \multirow[t]{3}{*}{ (Level) SC_T1 vs XM_T4 } & Observed count & 68 & 22 & 16 & 59 & 1631 \\
\hline & Expected count & 359.2 & 359.2 & 359.2 & 359.2 & 359.2 \\
\hline & Contribution to chi-square & 236.1 & 316.5 & 327.9 & 250.9 & 4503.0 \\
\hline \multirow[t]{3}{*}{ (Rising) SC_T2 vs XM_T2 } & Observed count & 55 & 17 & 21 & 68 & 1637 \\
\hline & Expected count & 359.6 & 359.6 & 359.6 & 359.6 & 359.6 \\
\hline & Contribution to chi-square & 258.0 & 326.4 & 318.8 & 236.5 & 4537.7 \\
\hline \multirow[t]{3}{*}{ (Low) SC_T3 vs XM_T1 } & Observed count & 625 & 165 & 102 & 149 & 754 \\
\hline & Expected count & 359 & 359 & 359 & 359 & 359 \\
\hline & Contribution to chi-square & 197.1 & 104.8 & 184.0 & 122.8 & 434.6 \\
\hline \multirow[t]{3}{*}{ (Falling) SC_T4 vs XM_T3 } & Observed count & 116 & 26 & 23 & 86 & 1539 \\
\hline & Expected count & 358 & 358 & 358 & 358 & 358 \\
\hline & Contribution to chi-square & 163.6 & 307.9 & 313.5 & 206.7 & 3896.0 \\
\hline
\end{tabular}

aNote: "1" stands for "completely different"; " 5 " stands for "completely the same". Values with statistical significance are shown in boldface.

Overall, participants were more likely to perceive SC_T3 and XM_T1 as the same tone, although they also gave slightly fewer but a comparable number of "different" responses.

\section{Falling contour: SC_T4 vs XM_T3}

The chi-square goodness-of-fit test showed that the responses were clearly not equally distributed $\left[\chi^{2}(4)\right.$ $=4887.59, p<0.001]$. The response category 5 contributed the most to the overall chi-square statistic, as demonstrated in Table VII. Pairwise comparisons showed that the frequency of the response category 5 was significantly higher than that of the other categories (all p's $<0.001$ ), indicating that SC_T4 and XM_T3 were mostly judged as 5, i.e., completely the same.

To summarize, the five-scale tone judgment results showed that the tone pair of level contour (SC_T1 and XM_T4) was mostly judged as the same by the XM_SC bidialectal speakers. Similarly, the tone pair of rising contour (SC_T2 and XM_T2) and the tone pair of falling contour (SC_T4 and XM_T3) were mostly judged as the same. The tone pair of low contour (SC_T3 and XM_T1) was differenct, which elicited a comparable number of "same" and "different" responses, although the two were statistically different. It seems that participants had a much harder time discriminating between the two tones of low contour in SC and XM.

\section{Comparisons among the four tone pairs}

Statistical results for the models of response showed a significant main effect of tone pair $\left[\chi^{2}(3)=253.69\right.$, $p<0.001]$, indicating that the rating tendency differed significantly among the four tone pairs (see Table VII). There was also a significant main effect of language order $\left[\chi^{2}(1)=22.61, p<0.001\right]$ and a significant main effect of speaker group $\left[\chi^{2}(1)=26.76, p<0.001\right]$. No effect of listener gender or interaction effect of the above factors was found (all $p$ 's $>0.05$ ). Specifically, when a XM tone was presented before its corresponding SC tone, listeners were more likely to rate higher, i.e., more likely to judge the two tones as being more alike $(\beta=0.35, z=5.08, p<0.001)$ compared to when a SC tone was presented before a XM tone. Likewise, listeners tended to rate higher for the male speaker group's speech than for the female speaker group's speech $(\beta=0.37, z=5.24, p<0.001)$.

Post hoc pairwise comparisons showed that the rating tendency of the tone pair of level contour was not significantly different from that of the tone pair of rising contour $(\beta=-0.002, z=-0.02, p=1.00)$. Both pairs were mostly judged as the same. Their rating tendencies, however, were significantly different from the tone pair of low contour (level vs low: $\beta=1.87, z=18.95, p<0.001$; rising vs low: $\beta=1.87, z=19.11, p<0.001)$ and the tone pair of falling contour (level vs falling: $\beta=0.31, z=3.08, p=0.01$; rising vs falling: $\beta=0.32, z=3.11, p=0.01)$. The rating tendencies of the tone pair of low contour and the tone pair of falling contour also showed a significant difference $(\beta=-1.55$, $z=-16.43, p<0.001)$. In summary, the rating tendency of the tone pair of low contour was significantly different from that of the other three tone pairs, with the former being judged as either different or the same (there were slightly more "completely the same" responses than "completely different" responses), whereas the latter three tone pairs were mostly judged as the same, although the tone pair of falling contour elicited more "different" responses than the tone pairs of level contour and rising contour.

\section{GENERAL DISCUSSION}

The present study investigated the phonological similarity in tones of two closely related Mandarin dialects, SC and $\mathrm{XM}$. Tones with similar contours from SC and XM were paired, and their acoustic properties were compared over properly controlled large samples produced by a group of highly proficient bidialectal speakers of XM and SC. F0 results of the four tone pairs ranged from no $\mathrm{F} 0$ difference (level contour tone pair) through F0 curvature difference 
(rising contour tone pair) to F0 height difference (falling contour tone pair) and F0 contour difference (low contour tone pair). Except for the falling contour tone pair, all the other tone pairs exhibited a difference in tone duration, and the largest duration difference was found in the low contour tone pair. These tone pairs of varying acoustic differences were then presented to the bidialectal speakers of XM and SC for tone perception with a five-scale tone judgment task. Results showed that the rating tendency of the tone pair of low contour was significantly different from that of the other three tone pairs, with the former being judged as either different or the same (there were slightly more "completely the same" responses than "completely different" responses), whereas the latter three tone pairs were mostly judged as the same, although the tone pair of falling contour elicited more "different" responses than the tone pairs of level contour and rising contour.

With a balanced comparable design, the present production and perception experiments empirically confirmed the systematic tonal mapping pattern between XM and SC proposed in Li (2001) and Zhang (2009). While there were detailed acoustic differences in tone production, tones with similar contours between the two dialects were basically perceived to be the same, resulting in mapped tone pairs of level contour (SC_T1 vs XM_T4), rising contour (SC_T2 vs XM_T2), and falling contour (SC_T4 vs XM_T3). Despite having distinct surface tonal contours, the tone pair of low contour (SC_T3 vs XM_T1) also showed mapping, although to a lesser degree compared to the other three tone pairs.

The mapping patterns of XM tones and SC tones were initially put forward based on the similarity of the tonal contour and pitch value of XM tones and SC tones represented on the five-point scale notation system (Chao, 1930, 1968). Different from the established pitch value of SC tones, there have been variances as to the specific pitch value of XM tones in previous studies (e.g., Bai, 1954; Luo and Wang, 1981; Ma, 2005; Peking University, 1989; Ren, 2012; Sun, 2007; Wang, 1996; Yuan, 1989; Zhang and Shi, 2009). However, the basic tonal contour shape of each tone was largely consistent across studies, and it has been noted that each XM tone has a mapped tone in SC with which it shares similar tonal contour and pitch value (Li, 2001; Zhang, 2009). Zhang (2009) tested the mapping pattern of the two tonal systems in tone production, but the tonal comparisons were not made on comparable datasets. The present study thus made more of an effort to empirically test the mapping pattern of the two tonal systems in tone production with a more balanced comparable design. Tonal comparisons were made on paired tones of similar tonal contours from the two dialects produced by highly proficient bidialectal speakers of SC and XM. Our acoustic results showed that except for the tone pair of level contour, all the other tone pairs showed difference in F0. Specifically, the XM rising tone had a shallower rising F0 curvature than the SC rising tone, with an overall comparable F0 mean. The XM falling tone had an overall lower F0 height than the SC falling tone. The XM low tone, not surprisingly, had a different F0 contour from the SC low-falling-rising tone. Our results of the specific F0 difference for each tone pair, except for the tone pair of low contour, was different from that found in Zhang (2009), showing that the manipulation of a comparable design in this study actually resulted in different tonal realizations. It is therefore important to test on comparable datasets in such cross-dialect investigations. Nevertheless, both studies showed an overall compact tonal space of XM tones than SC tones. Apart from the F0 difference, we also found a duration difference for each tone pair except for the tone pair of falling contour. All XM tones, other than the falling tone, tended to be shorter than their respective SC counterparts. On the whole, there were acoustic differences for each pair of tones with similar contours from SC and XM.

The acoustically different tone pairs, however, were mostly perceived to be the same or, at least, very similar by the bidialectal speakers of SC and XM. In a five-scale tone judgment task, the tone pairs of level contour, rising contour, and falling contour were mostly perceived to be completely the same, and the tone pair of low contour was also slightly more likely to be perceived as the same rather than different. Overall, these tone pairs of similar tonal contours from the two dialects were basically treated as the same during tone perception, despite the presence of acoustic differences. The results of our tone perception experiment confirmed the mapping pattern of XM tones and SC tones proposed in Li (2001) and Zhang (2009), providing new empirical evidence for the mapping of the two tonal systems from a perceptual point of view. Moreover, the mapping pattern seems to be more pronounced in tone perception than in tone production, given that each mapped tone pair was almost perceptually indistinguishable while having acoustically detectable differences.

The fact that the tone pairs of similar tonal contours from XM and SC were basically perceived to be the same by the bidialectal speakers does not mean that the participants did not pick up the acoustic differences at all. The mapped tone pairs did vary in the degree to which they were perceived as the same tones. The tone pairs of level contour and rising contour were mostly judged as the same by the bidialectal speakers of XM and SC, followed by the tone pair of falling contour. The tone pair of low contour elicited more "different" responses relative to the other three tone pairs. As only acoustic information was available to the participants during tone judgment, it is reasonable to assume that the different perceptual results for all the tone pairs resulted from their acoustic differences in some way. Our acoustic analyses demonstrated that the four tone pairs showed variance in different F0 dimensions, ranging from no F0 difference (level contour tone pair) through F0 curvature difference (rising contour tone pair) to F0 height difference (falling contour tone pair) and F0 contour difference (low contour tone pair). This varying acoustic difference in different F0 dimensions seems to have affected the tone perception results of each tone pair to varying degrees. Compared to the level contour tone pair with no F0 difference, the rising contour tone pair with F0 curvature 
difference was not perceived as any different, seemingly indicating that the bidialectal speakers of SC and XM were not sensitive to the F0 curvature difference between the two rising tones. This is not surprising, as F0 curvature has not been identified as a strong perceptual cue for tone discrimination. In contrast, the tone pair of falling contour with F0 height difference was perceived to be less similar than the tone pair of level contour with no F0 difference, suggesting that F0 height difference contributed to the discrimination of the two falling tones. This is consistent with the previous cross-language finding that F0 height is an important perceptual cue for tone discrimination (Gandour, 1983, 1984; Gandour and Harshman, 1978; Francis et al., 2008). Finally, the tone pair of low contour with F0 contour difference was perceived to be much more different than the tone pair of level contour with no F0 difference, as well as than the tone pair of falling contour with F0 height difference. Obviously, F0 contour difference significantly affected the discrimination between the two low tones. Also, the bidialectal speakers tended to be more sensitive to the dimension of F0 contour than F0 height in tone discrimination, as has been found by Gandour $(1983,1984)$ for SC speakers.

Note that although the duration property of each tone pair was maintained in the speech stimuli, participants did not seem to make full use of it in tone perception, if they used it at all. A duration difference was found in all the tone pairs except the falling tone pair. If the participants did use the duration cue for tone perception, with a duration difference of nearly $50 \mathrm{~ms}$, the tone pair of level contour, as well as the tone pair of rising contour, should have been judged as different tones rather than similar tones. If this is not convincing, a duration difference of about $166 \mathrm{~ms}$ in the tone pair of low contour should be certainly salient enough to rule out the possibility that the two tones were judged as similar. However, the pair of low contour tones ended up eliciting even slightly more "same" responses than "different" responses. Clearly, duration was not adopted as a valid perceptual cue for tone discrimination by the bidialectal speakers of SC and XM. They relied primarily on F0 information to make tone judgments.

Acoustic information, especially F0 information, is not the only perceptual cue that listeners employ during tone discrimination. Phonological rules can sometimes play a role in the process, too (Hao, 2012; Huang, 2012; So and Best, 2010). In this study, the tone pair of low contour (SC_T3 vs XM_T1) had distinct F0 contours. SC_T3 has a low-falling-rising contour and XM_T1 has a low-falling contour. If participants made tone perceptions purely based on acoustic information, the two low tones would have been judged as different. Instead, the two tones were perceived as either different or similar with even slightly more "same" responses than "different" responses. This could presumably be attributed to a phonological rule of SC_T3. SC_T3 has a low-falling-rising contour when it is in citation form or at the final position of an utterance. When placed before other tones or in a context, SC_T3 loses its rising tail and becomes a low-falling contour (Dow, 1972; Duanmu, 2007), which shows phonetic similarity to the XM low-falling tone. Participants seem to have applied this phonological rule of SC_T3 and used the context form SC_T3 to mediate between the citation form SC_T3 and XM_T1, and therefore perceived the citation form SC_T3 and XM_T1 as similar tones. Recall that we did not mention the source languages of the speech stimuli to the participants. It is therefore not clear where and how the context form SC_T3 came into play. There might be two scenarios. One is that $\mathrm{XM} \_\mathrm{T} 1$ here were considered as a representation of the context form SC_T3. Participants then made a comparison between the citation form SC_T3 with the context form SC_T3, which were judged as similar (citation form SC_T3 vs [XM_T1 $\rightarrow$ context form SC_T3]). The other scenario is that when presented with the citation form $\mathrm{SC}_{-} \mathrm{T} 3$ and XM_T1, participants activated the corresponding context form SC_T3, and they compared the context form SC_T3 with XM_T1, the latter being considered either as a representation of XM_T1 or context form SC_T3, resulting in a similar response ([citation form SC_T3 $\rightarrow$ context form SC_T3] vs XM_T1). In either scenario, the tone pairs of low contour should be judged as similar tones as a result of the phonological rule. In our result, the two low tones from SC and XM elicited a comparable number of "same" and "different" responses, suggesting that both the acoustic information and the phonological rule played roles in the tone discrimination process, and the two effects seem to counterbalance each other.

One might wonder whether the comparable number of "same" and "different" responses for the tone pair of low contour could be attributed to extreme individual variances, i.e., if some participants gave overwhelmingly more "different" responses than "same" responses or vice versa and shifted the overall perceptual pattern. In our data, almost all the participants gave both the "same" and "different" responses, indicating that they indeed used both acoustic cues and the phonological rule to make tone discrimination. There is no denying that the response distribution exhibited some differences among the participants. Some participants gave relatively more "different" responses than "same" responses, whereas others gave relatively more "same" responses than "different" responses. However, neither one was dominant. With the sample size like the one in this study, we believe that the individual differences were cancelled out, and our results reflected the interaction of the phonetic information and phonological knowledge during the tone discrimination process.

\section{CONCLUSION}

To conclude, the present study investigated the phonological similarity in tones of two closely related Mandarin dialects, SC and XM. Through production and perception experiments, it was established that there is systematic mapping of tones between XM and SC. The degree of similarity of the mapped tone pair in tone perception was largely dependent on the acoustic phonetic similarity between the tones in tone production, with a phonological rule playing a role in certain circumstance. 


\section{ACKNOWLEDGMENTS}

This research was supported by the Guangdong Planning Office of Philosophy and Social Science under Grant No. GD19YYY06 to M.L. and by the European Research Council (ERC) under the ERC Starting Grant (Grant No. 206198) to Y.C. We thank Dr. Xuhai Chen for providing access to the laboratory.

${ }^{1}$ Tones in modern Chinese were developed from those in Middle Chinese. There are four tonal categories in Middle Chinese, referred to as Ping, Shang, $Q u, R u$ in Chinese terms. Evidence suggests that three of the Middle Chinese tones, i.e., the Ping (level), Shang (rising), and $Q u$ (departing) tones have developed into the four modern tonal categories (Yinping, Yangping, Shangsheng, Qusheng) in a rather uniform way across dialects in the Mandarin family (Yuan, 1989). The $R u$ (entering) tone, characterized by syllables ending in stops, disappeared in most modern Mandarin dialects. These syllables with an entering tone have been distributed into the four modern Chinese tonal categories in different ways in different dialects. The four modern Chinese tonal categories Yinping, Yangping, Shangsheng, Qusheng are often labeled as T1, T2, T3, T4 nowadays. Note that a modern tonal category (e.g., T1) can have different tonal contours and pitch values in different Mandarin dialects.

${ }^{2} \mathrm{We}$ used $Z$-scores instead of $T$-values to normalize tone values because $T$ values can be easily distorted by extreme values like the maximum F0 or the minimum F0.

Bai, D. (1954). A Report on Guanzhong Dialect (Chinese Academy of Sciences Press, Beijing), Chap. 5, pp. 227-230 (in Chinese).

Bauer, R. S., and Benedict, P. K. (1997). Modern Cantonese Phonology (Mouton De Gruyter, Berlin), Chap. 2, pp. 162-164.

Boersma, P., and Weenink, D. (2015). "Praat: Doing phonetics by computer (version 5.4.21) [computer program]," http://www.praat.org (Last viewed March 15, 2020).

Chao, Y. R. (1930). “A system of tone letters,” Le Maitre Phonétique 45, 24-27. Chao, Y. R. (1968). A Grammar of Spoken Chinese (University of California Press, Berkeley), Chap. 1, pp. 25-30.

Chappell, H. (2001). Sinitic Grammar: Synchronic and Diachronic Perspectives (Oxford University Press, Oxford, UK), Chap. 1, pp. 3-28.

Cheng, C.-C. (1991). "Quantifying affinity among Chinese dialects," J. Chin. Linguist. 3, 76-110, available at http://www.jstor.org/stable/ 23827036.

Christensen, R. H. B. (2015). "Ordinal—Regression models for ordinal data ( $R$ package version 2015.6.28) [computer program]," http://www.cran.rproject.org/package $=$ ordinal/ $($ Last viewed March 25, 2020).

Czap, L., and Zhao, L. (2017). "Phonetic aspects of Chinese Shaanxi Xi'an dialect," in Proceedigs of the 8th IEEE International Conference on Cognitive Infocommunications (CogInfoCom 2017), Debrecen, Hungary, pp. 000051-000056.

$\mathrm{Da}, \mathrm{J}$. (2004). "A corpus-based study of character and bigram frequencies in Chinese e-texts and its implications for Chinese language instruction," in Proceedings of the 4th International Conference on New Technologies in Teaching and Learning Chinese, edited by P. Zhang, T. Xie, and J. Xu (The Tsinghua University Press, Beijing), pp. 501-511.

Dow, F. D. M. (1972). "A discussion on tone sandhi problems in Chinese," J. Int. Phon. Assoc. 2, 13-19.

Duanmu, S. (2007). The Phonology of Standard Chinese (Oxford University Press, Oxford), Chap. 10-11, pp. 225-274.

Francis, A. L., Ciocca, V., Ma, L., and Fenn, K. (2008). "Perceptual learning of Cantonese lexical tones by tone and non-tone language speakers," J. Phonetics 36, 268-294.

Gandour, J. T. (1983). "Tone perception in Far Eastern languages," J. Phonetics 11, 149-175.

Gandour, J. T. (1984). "Tone dissimilarity judgments by Chinese listeners," J. Chin. Linguist. 12, 235-261, available at http://www.jstor.org/stable/ 23767002

Gandour, J. T., and Harshman, R. A. (1978). "Crosslanguage differences in tone perception: A multidimensional scaling investigation," Lang. Speech 21, 1-33.
Guo, W., Yang, H., Liang, Q., and Pei, D. (2011). "Prosody conversion from Mandarin to Xi' an dialect," Comput. Eng. Appl. 47, 122-127 (in Chinese).

Hao, Y.-C. (2012). "Second language acquisition of Mandarin Chinese tones by tonal and non-tonal language speakers," J. Phonetics 40, 269-279.

Hashimoto, A. O.-K. Y. (1972). Phonology of Cantonese (Cambridge University Press, Cambridge, UK), Chap. 2, pp. 91-93.

Huang, T. (2012). "Cross-linguistic and inter-dialectal differences in tone perception by native speakers of three Chinese dialects and American English," J. Chin. Linguist. 40, 155-179, available at http://www.jstor. org/stable/23754202.

Kuznetsova, A., Brockhoff, P. B., and Christensen, R. H. B. (2017). "LmerTest package: Tests in linear mixed effects models," J. Stat. Softw. 82, 1-26.

Lam, A. (2005). "Language learning in China: The experience of four learners," Reflections Engl. Lang. Teach. 4, 1-14.

Lenth, R. V. (2016). "Least-squares means: The R package 1smeans," J. Stat. Softw. 69, 1-33.

Li, C. N., and Thompson, S. A. (1981). Mandarin Chinese: A Functional Reference Grammar (University of California Press, Berkeley), Chap. 1, pp. 1-2.

Li, D. C. S., and Lee, S. (2004). "Bilingualism in East Asia," in The Handbook of Bilingualism, edited by T. K. Bhatia and W. C. Ritchie (Blackwell, Malden, MA), pp. 742-779.

Li, P. (2001). "The correspondence pattern between Xi'an dialect and Putonghua in pronunciation," J. Xi'an Educ. Coll. 16, 57-61(in Chinese).

Li, R., and Stephen, W. (1987). Language Atlas of China (Longman, Hong Kong), p. B4.

Li, X. (2017). A Training Course on Mandarin Pronunciation, 2nd ed. (Communication University of China Press, Beijing), p. 2 (in Chinese).

Luo, C., and Wang, J. (1981). An Introduction to General Linguistics (The Commercial Press, Beijing), Chap. 5, p. 142 (in Chinese).

Liu, S., and Samuel, A. G. (2004). "Perception of Mandarin lexical tones when f0 information is neutralized," Lang. Speech 47, 109-138.

Ma, M. (2005). "Acoustic study of the tones of Xi'an dialect," J. Yanan Uni. (Social Science) 27, 110-112 (in Chinese).

Marian, V., Blumenfeld, H. K., and Kaushanskaya, M. (2007). "The language experience and proficiency questionnaire (LEAP-Q): Assessing language profiles in bilinguals and multilinguals," J. Speech Lang. Hear. Res. 50, 940-967.

Mirman, D. (2014). Growth Curve Analysis and Visualization Using $R$ (CRC Press, Boca Raton, FL), pp. 1-188.

Peking University (1989). A Dictionary of Pronunciations of Characters in Chinese Dialects (Wenzi Gaige Chubanshe, Beijing), p. 10 (in Chinese).

R Core Team (2015). "R: A language and environment for statistical computing [computer program]," https://www.r-project.org/ (Last viewed February 10, 2020).

Ren, J. (2012). "Investigations of Guangzhou, Jixi and Xi' an tones based on EGG," M.A thesis, Nanjing Normal University, Nanjing, China (in Chinese).

Rose, P. (1987). "Considerations in the normalisation of the fundamental frequency of linguistic tone," Speech Commun. 6, 343-352.

So, C. K., and Best, C. T. (2010). "Cross-language perception of non-native tonal contrasts: Effects of native phonological and phonetic influences," Lang. Speech 53, 273-293.

Sun L. (2007). A Study on Xi' an Dialect (Xi'an Publishing House, Xi'an), Chap. 1, p. 8 (in Chinese).

Wang, J. (1996). A Dictionary of Xi' an Dialect (Phoenix Publishing AND Media Network, Nanjing), p. 6 (in Chinese).

Whalen, D. H., and Xu, Y. (1992). "Information for Mandarin tones in the amplitude contour and in brief segments," Phonetica 49, 25-47.

Wiener, S., and Ito, K. (2015). "Do syllable-specific tonal probabilities guide lexical access? Evidence from Mandarin, Shanghai and Cantonese speakers," Lang. Cogn. Neurosci. 30, 1048-1060.

Wong, K. F., and Xiao, Y. (2010). "Diversity and difference: Identity issues of Chinese heritage language learners from dialect backgrounds," Heritage Lang. J. 7, 152-187.

Wu, J. (2015). "Tonal bilingualism: The case of two closely related Chinese dialects," Ph.D. dissertation, Leiden University, Leiden, The Netherlands.

Yang, R. (2015). "The role of phonation cues in Mandarin tonal perception,” J. Chin. Linguist. 43, 543-572. 
Yip, M. (2002). Tone (Cambridge University Press, Cambridge, UK), Chap. 7, pp. 171-211.

Yu, A. C. L. (2007). "Understanding near mergers: The case of morphological tone in Cantonese," Phonology 24, 187-214.

Yuan, J. (1989). An Introduction to Chinese Dialects, 2nd ed. (Wenzi Gaige Chubanshe, Beijing), Chap. 3-4, pp. 16-55 (in Chinese).
Zhang, J. (2009). "The comparative analysis of the monosyllabic tone between Xi' an and Beijing," J. Shaanxi Inst. Educ. 25, 71-75 (in Chinese).

Zhang, J., and Shi, F. (2009). "The statistical analysis on monosyllabic tone of Xi'an dialect," J. Xianyang Normal Univ. 24, 38-42 (in Chinese). 